\title{
Experimental investigation of layer-by-layer metallic photonic crystals
}

\author{
B. Temelkuran \\ H. Altug \\ E. Ozbay
}

Indexing terms: Photonic crystals, Defect structures, Millimetre waves, Resonant detectors

\begin{abstract}
The authors have investigated the transmission properties and defect characteristics of layer-by-layer metallic photonic crystals. Thay have demonstrated experimentally that the metallicity gap of these crystals extends to an upper band-edge frequency, and no lower edge was detected down to $2 \mathrm{GHz}$. The defect structures built around these crystals exhibited high transmission peak amplitudes $(100 \%)$ and high $Q$ factors (2250). The crystals with low filling ratios (around 1-2\%) were tested and were still found to possess metallic photonic crystal properties. These crystals exhibited high reflection rates within the metallicity gap and reasonable defect mode characteristics. A power enhancement factor of 190 was measured for the electromagnetic (EM) wave within planar cavity structures, by placing a monopole antenna inside the defect volume. These measurements show that detectors embedded inside a metallic photonic crystal can be used as frequency selective resonant cavity enhanced (RCE) detectors with increased sensitivity and efficiency when compared to conventional detectors.
\end{abstract}

\section{Introduction}

Photonic crystals are periodic structures having the property of reflecting the EM waves in all directions for a certain range of frequencies [1-6]. Earlier investigations were mainly concentrated on dielectric based photonic crystals, and were successful in various applications [7-10] but some of their properties restrict the wide usage of these materials. First, dielectric based photonic crystals have low rejection rates per layer, so a large number of layers are needed for applications requiring high isolation purposes [11]. Second, for applications around the $1-10 \mathrm{GHz}$ range, these crystals become impractical due to their relatively large dimensions. As an exaniple, for a $2 \mathrm{GHz}$ application, the dielectric based photonic crystal will have a typical size of $100 \times 100 \times 20 \mathrm{~cm}$, which will be an important limiting

(C) IEE, 1998

IEE Proceedings online no. 19982471

Paper first received 25th June 1998 and in revised form 18th November 1998

The authors are with the Department of Physics, Bilkent University, Bilkent, Ankara, 06533 Turkey factor for many applications. These problems can be solved by introducing metals to photonic crystals [12-21].
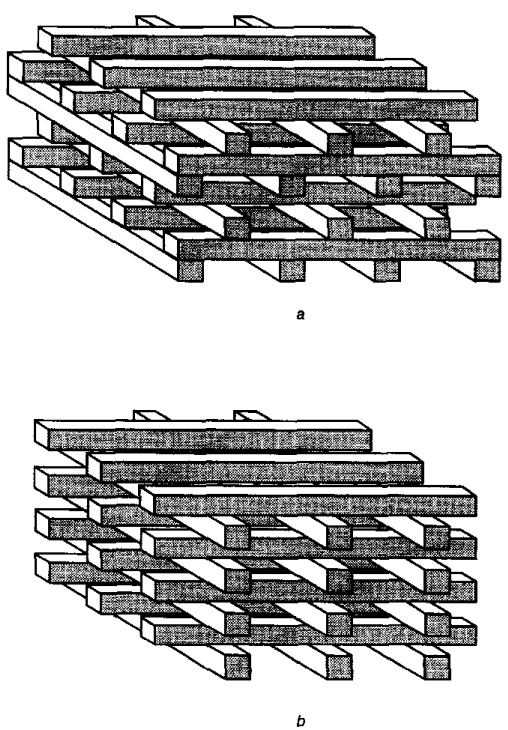

Fig. 1 Schematic diagrams of fct and st based photonic crystals $a$ fct; $b$ st based crystals

\section{Crystal structure}

In this paper, we have investigated the properties of two types of layer-by-layer metallic photonic crystals. The first structure, in which the stacking sequence repeats every four layers, is the equivalent of a face centred tetragonal (fct) lattice as shown in Fig. $1 a$. Fig. $1 b$ shows the other structure similar to the first, but with a repeating sequence of two layers, which is the analogue of a simple tetragonal (st) lattice. The metallic rods used in these crystals were $0.8 \mathrm{~mm}$ wide, $2.5 \mathrm{~mm}$ thick and $120 \mathrm{~mm}$ long, and were placed with a centre to centre separation of $7.6 \mathrm{~mm}$. The rods were obtained by machining $150 \times 150 \times 5 \mathrm{~mm}$ aluminium blocks. These blocks were then stacked together to form either fct or st structures depicted in Fig. 1.

\section{Transmission properties of metallic photonic crystals}

We first measured the transmission properties of both structures using a Hewlett-Packard $8510 \mathrm{C}$ network analyser, and standard-gain microwave antennas. Fig. $2 a$ compares the transmission properties of 6-layer 
fct and st based crystal structures where the propagation direction of the EM wave is along the stacking direction. Both crystals yielded bandgaps with upper edges $\sim 20 \mathrm{GHz}$, and no lower edge exists down to 10 GHz. Fig. $2 a$ shows the rejection ratios for two different crystal structures at $12 \mathrm{GHz}$, as the number of layers are increased. The typical rejection factors of 7-8 dB per layer are observed at $10 \mathrm{GHz}$, which is significantly superior to dielectric-based photonic crystals having a maximum rejection of $3-4 \mathrm{~dB}$ per layer at midgap frequencies.

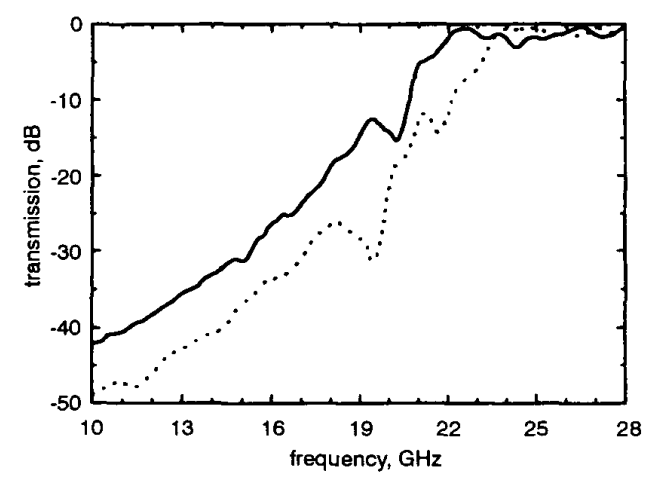

a

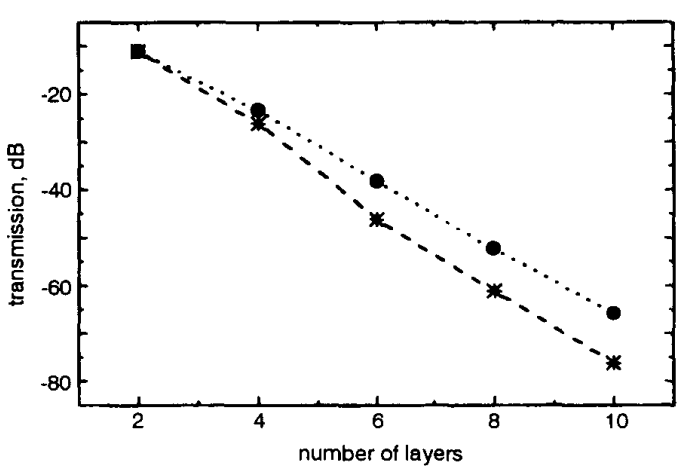

$b$

Fig.2 Transmission characteristics of 6-layer fat and st based metallic photonic crystals, and change in rejection rates of these crystal structures

as number of layers is increased

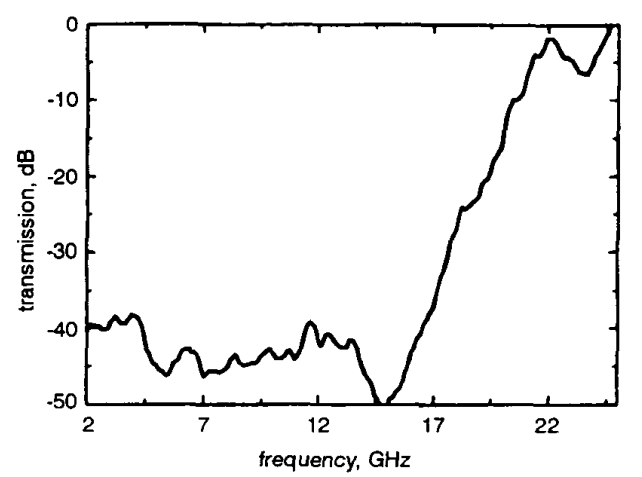

Fig.3 Transmission measurement performed by monopole antennas show that metallicity gap extends down to lower frequencies

The metals are peffect reflectors at microwave frequencies, and EM waves cannot penetrate into these materials. This property results in the metallicity gap which is expected to extend down to $0 \mathrm{GHz}$ [19]. In order to verify this, we measured the transmission properties of a 10-layer st based metallic crystal using two monopole antennas, instead of standard gain horn antennas. The monopole antennas, which were constructed by removing the shield around one end of a microwave coaxial cable, were used to transmit and receive the EM wave radiation. As seen in Fig. 3, the bandgap still exists even at frequencies as low as $2 \mathrm{GHz}$. Since the monopole antenna radiates in all directions, these measurements also reveal the fact that the metallicity gap conserves its property of reflecting the EM wave in all directions. The relatively low rejection ratio observed, which is still higher than its dielectric equivalent, is mainly due to the following two reasons: first, the finite size of our crystal compared to large wavelengths at which the measurement is made was a limiting factor. Secondly, the radiation efficiencies of the monopole antennas used were relatively poor.

As we mentioned earlier, if dielectric materials were used, the crystal dimensions will be in the metre scale in order to have a bandgap at $2 \mathrm{GHz}$. The dimensions of the metallic crystal we investigated, which is suitable for applications at these frequencies, is about an order of magnitude smaller than its dielectric counterpart. The three orders of magnitude reduction in the structure volume makes the usage of metallic photonic crystals feasible for low frequency applications.

\section{Defect structures in metallic photonic crystals}

It was previously shown in dielectric based layer-bylayer photonic crystals that defect structures around this geometry can be built by means of adding or

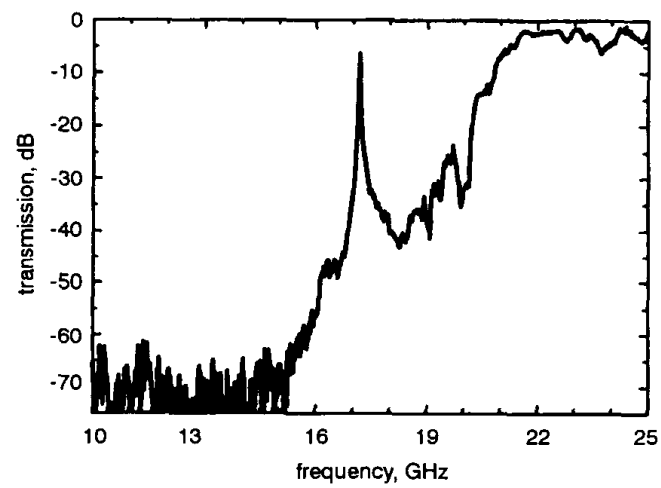

a

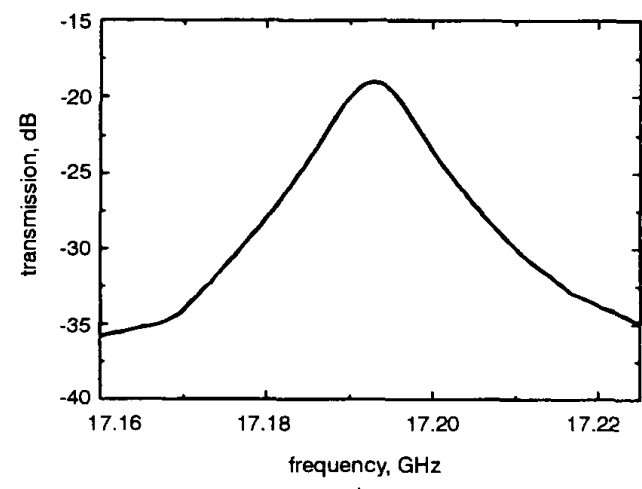

Fig.4 Transmission characteristics of 14-layer st based crystal with a single rod removed defect structure, and expanded frequency scale of a similar defeet structure built around 18-layer crystal 
removing rods from an otherwise perfect crystal [11]. The same idea was used to investigate the defect characteristics of these metallic photonic crystals [12]. Fig. $4 a$ shows the transmission properties of 14-layer st type photonic crystals where the 7th layer is the defect layer with a single missing rod. The defect mode was only observed when the electric field polarisation vector of the incident EM wave $e$ was parallel to the rods of the defect layer. The defect mode was observed at $17.2 \mathrm{GHz}$ with a peak transmission amplitude of $-7 \mathrm{~dB}$, and a $Q$ factor (quality factor, defined as the centre frequency divided by the peak's full width at half maximum) of 750 . Increasing the number of layers to 18 (where the 9th layer was chosen as the defect layer) yielded a defect mode with an increased $Q$ factor of 1740 and a peak transmission of $-19 \mathrm{~dB}$ (Fig. $4 b$ ).

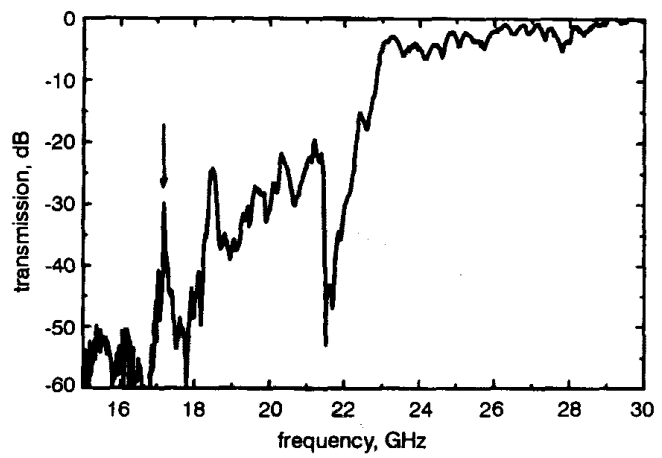

Fig.5 Defect characteristics of a 14-layer fct based crystal with a single rod removed defect structure

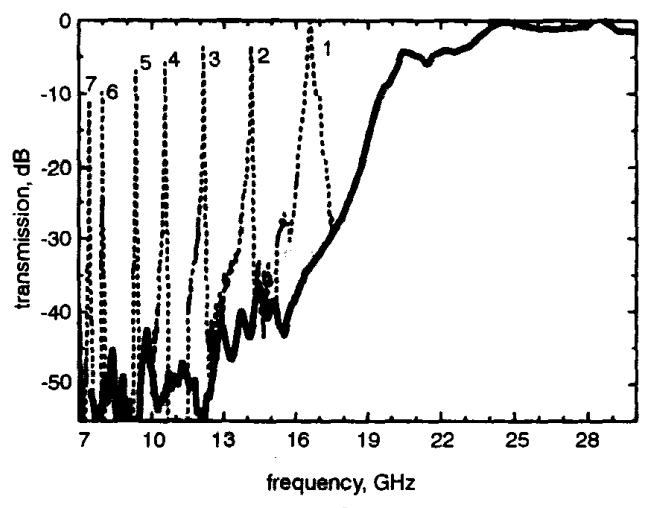

a

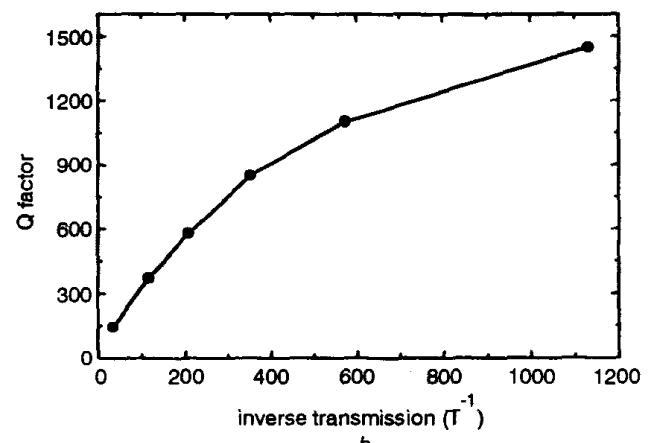

$b$

Fig.6 Transmission characteristics of 8-layer st based photonic crystal with no gap (solid line), and with planar defects with separation widths of with no gap (ii) $5 \mathrm{~mm}$, (iii) $7 \mathrm{~mm}$, (iv) $9 \mathrm{~mm}$, (v) $11 \mathrm{~mm}$, (vi) $14 \mathrm{~mm}$ and (vii) $15.5 \mathrm{~mm}$ (dotted lines), and change in $Q$ factor of defect modes as a function of inverse mirror transmission of cavity at defect frequency
A similar defect structure built around fct based photonic crystal gave a higher $Q$ factor of 950 , but with a low peak transmission amplitude of $-30 \mathrm{~dB}$ (Fig. 5). This put a limit to achieve a higher $Q$ factor by increasing the number of layers, where the peak transmission dropped below the noise level. In the following investigations, we concentrated on st based metallic photonic crystals, which are superior to fet based photonic crystals considering the advantages of the higher peak amplitude and maximum achievable $Q$ factors.

We then investigated the planar type of defect structures, built around an 8-layer st based photonic crystal. The planar defect was obtained by separating the 4 th and 5th layers of the crystal. This resulted in a planar airgap between the two photonic mirrors, each formed of a 4-layer (2-unit cell) crystal. Fig. $6 a$ shows the transmitted EM wave, along the stacking direction, through the planar defect structure with separation widths of $3,5,7,9,11,14$ and $15.5 \mathrm{~mm}$ (with corresponding labels $1,2,3,4,5,6$ and 7 , dotted lines), and with no gap (solid line). Although the transmitted peak amplitude drops from $100 \%$ transmission at $17 \mathrm{GHz}$ to $-11.3 \mathrm{~dB}$ at $7.4 \mathrm{GHz}$, there exists a significant increase in the $Q$ factor from 115 to 1450 . Both of these observations can be explained by considering our structure as a Fabry-Perot cavity. The increase in the reflectivities of the mirrors of a Fabry-Perot cavity results in a lower transmitted peak amplitude and a higher $Q$ factor [22]. In order to see this effect, we plotted the $Q$ factors of the defect modes as a function of inverse transmission of the mirrors (4-layer crystal) of the cavity at the corresponding defect frequency (Fig. $6 b$ ). The curve is expected to be linear if the mirrors of the cavity were large compared to the resonant wavelength of the cavity [22]. As the resonant wavelength and the cavity width increases, due to the finite size of our crystal mirrors, the curve deviates from linearity and flattens. These results show that this structure can be used to create defect modes with high $Q$ factors at frequencies as low as a few $\mathrm{GHz}$, which is nearly impossible for dielectric based photonic crystals.

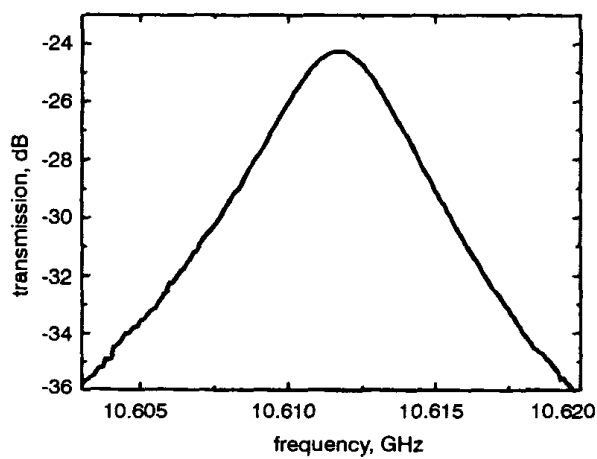

Fig. 7 Transmission characteristics of a planar defect mode with a $Q$ factor of 2250, in an expanded frequency scale

Another way to increase the $Q$ factors of these planar defect structures is to increase the rejection ratio of photonic mirrors of the cavity by increasing the number of layers. As shown in Fig. 7, a 12-layer photonic crystal separated from the middle with a separation width of $10 \mathrm{~mm}$ resulted in a $Q$ factor of 2250 , with a peak transmission amplitude of $-25 \mathrm{~dB}$ at $10.6 \mathrm{GHz}$. 
We continued to explore the properties of the planar defects in metallic structures by changing the angle of incidence of the EM wave. Increasing the angle of incidence, peaks other than the main peak at $\theta=0^{\circ}$ (where $\theta$ is the angle between the surface normal of the crystal and the incident EM wave) came into view. If we continue to increase the angle further, we observe that a number of peaks appearing at higher frequencies dominate, while the peak transmission amplitudes of lower
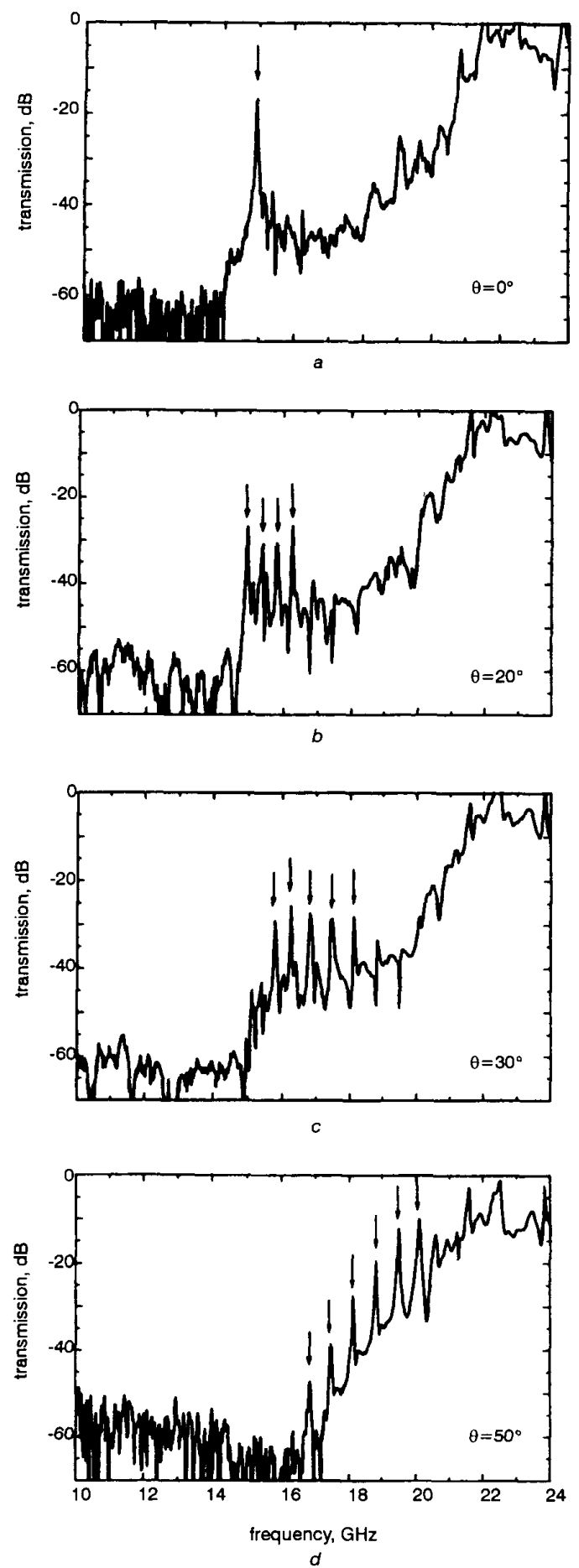

Fig.8 Additional defect modes observed for planar defect structures with increasing angle of incidence frequency modes diminish, disappearing at the noise level. The frequencies of the defect modes do not shift with the changing angle of incidence, but dominate at certain fixed frequencies for certain angles. These observations are demonstrated in Fig. 8, where the angle of incidence is changed from $0^{\circ}$ to $50^{\circ}$, measured from a 12-layer photonic crystal separated from the middle by a $5 \mathrm{~mm}$ gap. These defects cannot be explained by a simple Fabry-Perot model $[11,13]$, since the next resonance is around $7-8 \mathrm{GHz}$ away from the observed one, while the consecutive peaks appear with a separation < 1GHz. An explanation can be made using the theory of resonant cavities, where the reflecting mirrors are considered to be curved. The solution for this structure is a combination of Hermite Polynomials, which results in additional peaks other than the main resonance. This unexpected phenomenon is under further theoretical and experimental investigation.

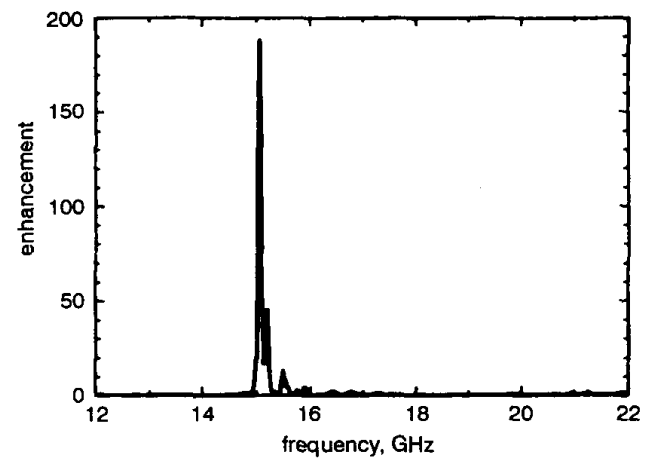

a

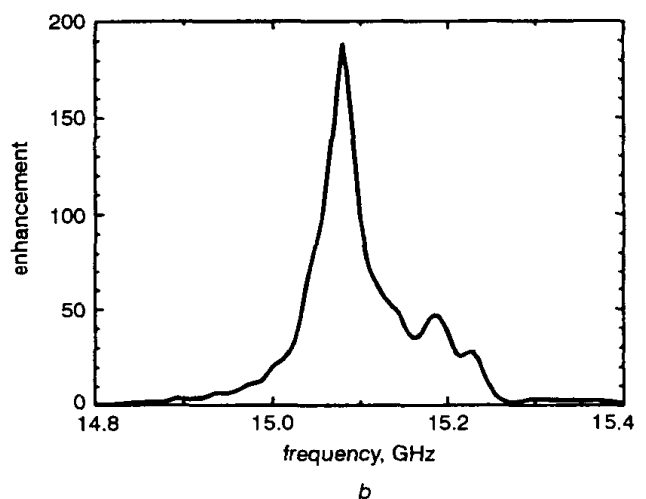

Fig.9 Enhancement characteristics of a planar defect structure within 8-layer st based metallic photonic crystal

\section{Resonant cavity enhancement}

The electric fields in cavities are usually enhanced, and by placing active devices in such cavities, one can make the device benefit from the wavelength selectivity and the large enhancement of the resonant EM field within the cavity [22]. This effect has already been used to achieve novel devices such as resonant cavity enhanced (RCE) photodetectors and light emitting diodes $[23,24]$. In order to demonstrate this effect, we placed a monopole antenna inside the defect volume of planar defect structures described above. We used the network analyser and the standard gain horn to obtain EM waves, and the monopole antenna, which is also connected to the network analyser, as a receiver. The sin-

IEE Proc:-Optoclectron. Vol. 145. No. 6. December 1998 
gle pass absorption data of the probe was used to calibrate the measured enhancements.

Our previous investigations on dielectric photonic crystals show that asymmetric planar cavities result in higher enhancement factors when compared to symmetric ones [25]. Fig. 9 shows the enhancement characteristics of a planar type of defect structure, with a 4layer thick front mirror and a 6-layer thick back mirror. At a $5 \mathrm{~mm}$ separation width of the cavity, the defect frequency was observed to be at $15.08 \mathrm{GHz}$. We observed a power enhancement factor of 190 at the defect frequency, with a $Q$ factor of 335 . When compared with enhancement factors obtained in cavity structures built around dielectric photonic crystals, this value is rather small. This may be the result of small but finite absorption coefficients of metals, which exist even at microwave frequencies. This absorption becomes significant when we consider the high number of times the field circulates inside the cavity and experiences a loss in each cycle. Still these investigations suggest the possibility of using an embedded detector inside a metallic photonic crystal, as a frequency selective RCE detector with an increased sensitivity and efficiency. As an example, detectors based on photonic crystals will be more efficient than a typical frequency selective $L C$ resonant circuit detector as the EM field does not recycle in such detectors. The RCE detectors based on photonic crystals are also superior to other types of resonant cavity antennas such as patch antennas. The ultra-high EM wave rejection properties of photonic crystals result in better and more controlled confinement of the resonant EM field in all directions. This means photonic crystal based RCE detectors will have higher quality factors and better frequency selective properties when compared to conventional $\mathrm{RCE}$ detectors.

\section{Towards lower filling ratios}

Although the filling ratio of the metallic crystal we have investigated has quite a lower filling ratio $(11 \%)$ when compared with its dielectric equivalent $(30 \%)$, considering the high rejection ratio property of metals, it is possible to lower the filling ratio further. Therefore, we constructed and tested a new layer-by-layer structure, with cylindrical metallic rods which are $0.7 \mathrm{~mm}$ in radius, $150 \mathrm{~mm}$ long, and with centre to centre separation of $6 \mathrm{~mm}$. The rods of the consecutive layers are placed perpendicular to each other, along the stacking direction, and are touching in couples. These touching couples of layers form a square mesh surface, and are separated from each other by $6 \mathrm{~mm}$ in st and $3 \mathrm{~mm}$ in fet based crystals. This structure results in a filling ratio of $1.1 \%$ in st and $2.2 \%$ in fet crystals.

Fig. 10 shows the transmission properties of 7-mesh layer fct $(a)$ and st $(b)$ based photonic crystals. The upper band-edge is at $24 \mathrm{GHz}$ in fet based crystal, and shifts to $15 \mathrm{GHz}$ in st based crystal. The metallicity gap extends to lower frequencies as expected. The average rejection rate per mesh layer of these crystals is $\sim 8$ $10 \mathrm{~dB}$, which is superior to that of the dielectric based photonic crystals.

Defects created by removing rods in this structure resulted in similar defect modes observed in other photonic crystals, but with lower peak transmission amplitudes and lower $Q$ factors. Fig. 11 shows the transmission properties of a 7-mesh layer fet $(a)$ and st (b) based crystals with a defect introduced by removing
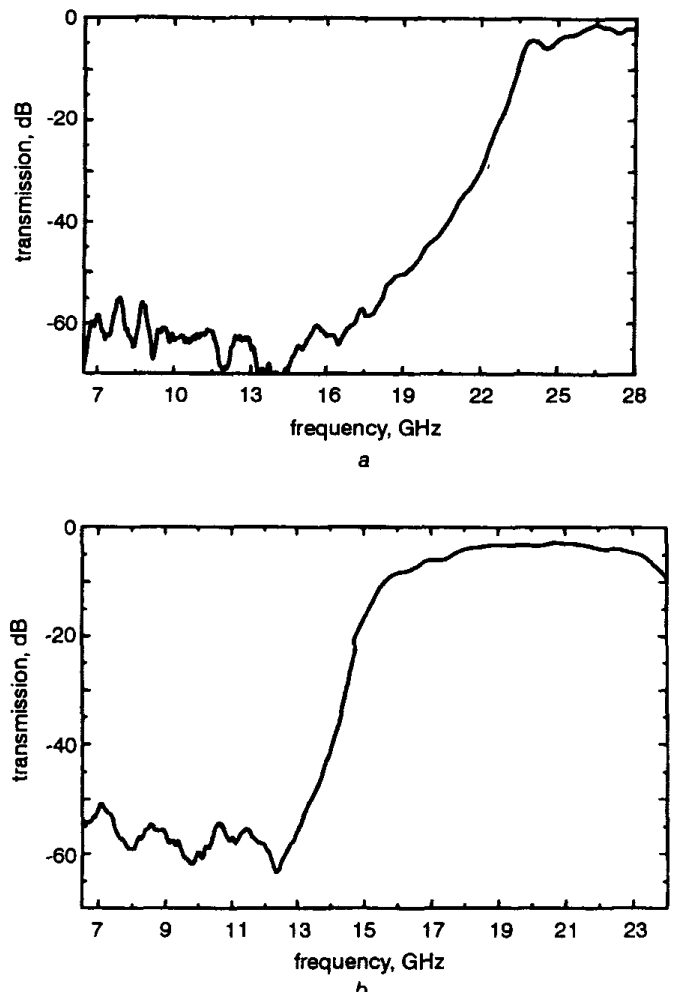

Fig.10 Transmission properties of low filling ratio $f c t$ and st based layer-by-layer metallic photonic crystals $a y e r-b y$-layer metallic
$a$ fet; $b$ st based crystals
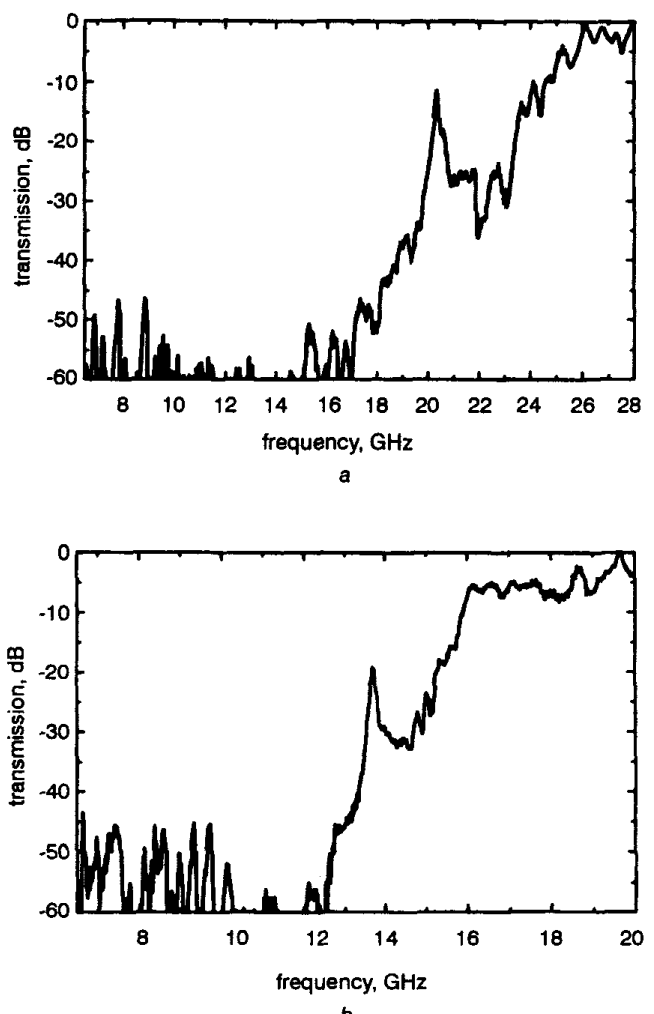

Fig. 11 Characteristics defect structures built around fct and st based low filling ratio photonic crystals $a$ fct; $b$ st based crystals 
a single rod from the 4th layer. This time, contradictory to the other type of crystal, fet based crystal is superior to st based one considering the higher peak transmission amplitudes $(-11.2 \mathrm{~dB}$ in fet and $-18.4 \mathrm{~dB}$ in st) along with the same $Q$ factors (150 in both). The relatively poor defect characteristics of st based crystals may be assigned to the leakage of the EM waves from the sides of the separation planes of the consecutive touching couples of layers, which is lower (but still significant) in fet based crystals.

These results, although not as good as the previous type of crystal, are of great importance considering applications which require crystals that are light and that have low filling ratios. Also, since metals exhibit high absorption at optical frequencies, these crystals with their minimised metal contents, when compared to the other metallic crystals we investigated, will be quite useful at optical frequencies if built in sufficiently small dimensions.

\section{Conclusion}

We have investigated the properties of metallic layerby-layer photonic crystals. An average rejection rate of $8 \mathrm{~dB}$ per layer was measured. We have observed that the metallicity gap extends down to frequencies as low as $2 \mathrm{GHz}$ without any lower band-edge. Defect modes created by removing rods resulted in high peak transmission $(80 \%)$ and high $Q$ factors (1740). Planar type defect structures exhibited $100 \%$ peak transmission amplitudes, and higher $Q$ factors (2250), and existed at low frequency values $(\sim 7 \mathrm{GHz})$. We also demonstrated the RCE effect in planar cavities built around metallic photonic crystals. A new type of crystal with very low filling ratio is tested and the existence of the metallicity gap and the defect modes were observed. With all these advantages, metallic photonic crystals are suitable for various applications at microwave, millimetre wave and submillimetre wave frequencies. Considering the second type of crystal (with low filling ratio), these applications may also be extended to optical frequencies.

\section{Acknowledgments}

This work is supported by the Turkish Scientific and Technical Research Council of Turkey (TÜB I TAK) under contract 197-E044, NATO Grant SfP971970, National Science Foundation Grant INT-9512812 and NATO-Collaborative Research Grant 950079.

\section{References}

1 HO, K.M.. CHAN, C.T., and SOUKOULIS, C.M.: 'Existence of a photonic gap in periodic dielectric structures', Phys. Rev. Lett., $1990, \mathbf{6 5},(25)$, pp. $3152-3155$

2 YABLONOVITCH, E., GMITTER, T.J., and LEUNG, K.M.: 'Photonic band structure: the face-centered-cubic case employing nonspherical atoms', Phys. Rev. Lett., 1991, 67, (17), pp. 22952298

3 JOHN, S.: 'Strong localization of photons in certain disordered dielectric superlattices', Phys. Rev. Lett., 1987, 58, (23), pp. 2486 2489
4 SOUKOULIS, C.M. (Ed.): 'Photonic band gap materials' (Plenum, New York, 1996)

5 JOANNOPOULOS, J.D.. VILLENEUVE, P.R., and FAN, S.: 'Photonic crystals: putting a twist on light', Nature, 1997, 386, pp. 143-149

6 KRAUSS, T.F., DE LA RUE, R.M., and BRAND, S.: 'Twodimensional photonic-band gap structures operating at near-infrared wavelengths', Nature, 1996, 283, pp. 699-702

7 OZBAY, E.: 'Layer-by-layer photonic crystals from microwave to far infrared frequencies, $J$. Opt. Soc. $A m . B, 1996,13,(9)$, pp. $1945-1955$

8 WANKE, M.C., LEHMANN, O., MULLER. K., WEN. Q.. and STUKE, M.: 'Laser rapid prototyping of photonic band-gap microstructures', Science, 1997, 275, (5304), pp. 1284-1286

9 HO, K.M., CHAN, C.T., SOUKOULIS, C.M., BISWAS, R., and SIGALAS, M.: 'Photonic bandgaps in three dimensions: New layer-by-layer periodic structures'. Solid State Commun., 1994, 89, (5), pp. 413-416

10 KRAUSS, T.F., VÖGELE, B., STANLEY, C.R., and DE LA RUE, M.: 'Waveguide microcavity based on photonic microstructures', IEEE Photonics. Technol. Lett., 1997, 9, (2), pp. 176-178

11 OZBAY, E., and TEMELKURAN, B.: 'Reflection properties and defect formation in photonic crystals', Appl. Phys. Lett., $1996,69,(6)$, pp. $743-745$

12 OZBAY, E., TEMELKURAN, B., SIGAlAS, M., TUTTLE, G., SOUKOULIS, C.M., and HO, K.M.: 'Defect structures in metallic photonic crystals', Appl. Phys. Lett., 1996, 69, (25), pp. 3797-3799

13 TEMELKURAN, B., OZBAY, E., SIGALAS, M., TUTTLE, G., SOUKOULIS, C.M., and HO, K.M.: 'Reflection properties of metallic photonic crystals', Appl. Phys. A, 1998, 66, pp. 363365

14 JOANNOPOULOS, J.D. MEADE, R.D., and WINN, J.N. 'Photonic crystals' (Princeton University Press, 1995)

15 SIEVENPIPER, D.F. YABLONOVITCH, E., WINN, J.N., FAN, S., VILLENEUVE. P.R., and JOANNOPOULOS, J.D.: 3D metallo-dielectric photonic crystals with strong capacitive coupling between metallic islands', Phys. Rev. Lett., 1998, 80, (13), pp. 2829-2832

16 GUPTA, S., TUTTLE, G., SIGAlAS, M., and HO, K.M.: 'Infrared filters using metallic photonic bandgap structures on flexible substrates', Appl. Phys. Lett., 1997, 71, (17), pp. 2412 2414

17 KUZMIAK, V., and MARADUDIN, A.A.: 'Photonic structures of one- and two-dimensional periodic systems with metallic components in the presence of dissipation", Phys. Rev. B, 1997, 55 (12), pp. 7427-7444

18 SCALORA, M., BLOEMER, M.J., PETHEL, A.S., DOWLING. J.P., BOWDEN, M., and MANKA, A.S.: 'Transparent, metallodielectric, one dimensiomal, photonic band-gap structures', $J$. Appl. Phys, 2377-2383, 1998, (83), pp. 5

19 SIEVENPIPER, D.F., SICKMILLER, M.E., and YABLONOVITCH, E.: '3D wire mesh photonic crystals', Phys. Rev. Lett., $1996,76,(14)$, pp. $2480-2483$

20 MCINTOSH, K.A., MAHONEY, L.J., MOLVAR, K.M. MCMAHON, O.B., VERGHESE, S., RÖTHSCHILD, M., and BROWN, E.R.: "Three-dimensional metallodielectric photonic crystals exhibiting resonant infrared stop bands', Appl. Phys. Lett., 1997, 70, (22), pp. 2937-2939

21 SIGALAS, M.M CHAN, C.T HO, KM and SOUKOULIS, C.M.: 'Metallic photonic band-gap materials', Phvs. Rev. $B, 1995,52,(16)$, pp. 11744-11751

22 SIEGMAN, A.E.: 'Lasers' (University Science Books, Mill Valley, 1986)

23 UNLU, M.S., GOKKAVAS, M. ONAT, B.M., ATA, E.. OZBAY, E.. MIRIN, R.P., KNOPP, K.J. and CHRIS TENSEN, D.H.: 'High bandwidth-efficiency resonant cavity enhanced Schottky photodiodes for $800-850 \mathrm{~nm}$ wavelength operation', Appl. Phys. Lett., 1998, 72, (2), pp. 2727-2729

24 OZBAY, E. ISLAM M.S ONAT B GOKKAVAS M AYTUR, O., TUTTLE, G., TOWE, E., HENDERSON, R.H., and UNLU, M.S.: 'Fabrication of high-speed resonant cavity
enhanced schottky photodiodes', IEEE Photonics. Technol. Lett., enhanced schottky photo
$1997,9,(5)$, pp. $672-674$

25 TEMELKURAN, B., OZBAY, E, KAVANAUGH, J.P., TUTTLE, G., and HO, K.M.: 'Resonant cavity enhanced detectors embedded in photonic crystals', Appl. Phys. Lett., 1998, 72, (19), pp. $2376-2378$ 